\title{
Focus group discussions to inform the design of a weight loss programme suitable for overweight and obese women treated for breast cancer
}

\author{
R. Newlands ${ }^{1}$, J. Clark ${ }^{1}$, L. C. A. Craig ${ }^{1}$, G. McNeill ${ }^{1}$, P. Hoddinott ${ }^{2}$ and S. Heys ${ }^{3}$ \\ ${ }^{1}$ Public Health Nutrition Research Group, University of Aberdeen, Aberdeen AB25 2ZD, ${ }^{2}$ Health Services Research Unit, \\ University of Aberdeen, Aberdeen AB25 2ZD and ${ }^{3}$ Division of Applied Medicine, University of Aberdeen, Aberdeen
}

$A B 252 Z D$

Women who are overweight or obese at the time of breast cancer diagnosis and/or who gain weight after diagnosis are at higher risk for adverse clinical outcomes ${ }^{(1)}$. Therefore, improving the quality of life and reducing the risk of recurrence for this population group has become very important ${ }^{(2)}$. Only a few interventions have been conducted targeting weight loss for women already treated for breast cancer $^{(3)}$. The present study used qualitative research to inform the design of a weight loss intervention for women treated for breast cancer.

As part of the development of the intervention, 15 women were recruited from a local female cancer support charity, who had previously undergone treatment for breast cancer. Three focus group discussions were held, led by a dietitian (JC) in a local cancer support centre. Topics addressed in the focus groups included post diagnosis weight issues, eating and exercise patterns, barriers to maintaining a healthy weight, and their past experience of and future preferences for a weight loss programme. All meetings were recorded and transcribed verbatim by a trained typist. The data was analysed using Framework analysis. The protocol was approved by the North of Scotland Research Ethics Committee.

Analysis of the data revealed that majority of the women had put on weight during their treatment and/or post treatment. The most commonly reported causes for their weight gain were lifestyle changes, side-effects of treatments and the menopause, though some women thought it was difficult to isolate the cause of their weight gain. Although a number of women had tried different diets and joined various health clubs and slimming clubs, they discussed a number of barriers towards weight loss including lack of energy and selfesteem, lack of knowledge and self-motivation, lack of guidance from health professionals and disability due to arm, hip and muscle pain. But many would have liked to join a weight loss programme to improve their self-confidence. Their preference was for a programme delivered three to six months after completion of hospital treatment either in a group of women treated for breast cancer or one-to-one sessions followed by a small group. They would have liked this programme to be delivered in a non-clinical setting e.g. a cancer support centre or a community centre. Their preference was for gentle exercises such as slow dance with music, yoga, walking, swimming and also any healthy dietary advice which would stop the cancer recurring.

The findings from these focus group discussions, along with results from a questionnaire survey in a larger more representative sample informed from these discussions, will aid in the design of a weight loss intervention suitable for women treated for breast cancer.

Funded by Fraserburgh Moonlight Prowl and CRANES (Cancer Research Aberdeen and North East Scotland)

1. WHO (2006) World Health Organisation: Guidelines for Management of Breast Cancer.

2. WCRF (2011) Updating the evidence on breast cancer survivors, World Cancer Research Fund, London http://www.wcrfuk.org/cancer_prevention/ health_professionals/informed_articles/evidence breast_cancer_survivors.php

3. Murray D (2009) Weight Management Interventions for Overweight and Obese Breast Cancer Survivors: Systematic Literature Review; BSc Project, University of Aberdeen. 